\title{
A multi-purpose phantom kit for magnetic particle imaging
}

\begin{abstract}
Phantoms are essential tools for the development and characterization of Magnetic Particle Imaging (MPI), an imaging technique that can quantitatively map the spatial distribution of magnetic nanoparticles (MNP). The objective of this study was to develop and validate a modular MPI phantom kit with high versatility for platform-independent quality assurance and the assembling of defined geometries in MPI. It was shown that the developed MPI phantom kit can be used for both application scenario testing and quality assurance in MPI which provides the basis for future reference phantoms to directly compare existing scanners within the MPI community.
\end{abstract}

Keywords: additive manufacturing, 3D printing, phantoms, medical imaging, magnetic particle imaging

https://doi.org/10.1515/cdbme-2021-2081

\section{Introduction}

Phantoms are essential tools for the development and characterization of imaging techniques. They represent defined shapes and properties to investigate image resolution, sensitivity, or geometric distortions. Furthermore, they are used for training purposes to simulate application scenarios. Phantoms are also mandatory for Magnetic Particle Imaging (MPI), an imaging technique that can quantitatively map the spatial distribution of magnetic nanoparticles (MNP) [1,2]. Currently, the potential of MPI is being explored in relevant application areas through intensive research and development work. For MPI imaging, scanners with different imaging concepts are used, which thus differ in their spatial dimensions and technical specifications. In addition to the classic coil design with cylindrical bore, for example, there are also open

*Norbert Löwa, Dirk Gutkelch, Olaf Kosch, Frank Wiekhorst: Physikalisch-Technische Bundesanstalt (8.23 Metrology for Magnetic Nanoparticles), Berlin, Germany, norbert.loewa@ptb.de Rebecca Hoffmann, Silvio Dutz: TU IImenau (Fakultät für Informatik und Automatisierung, Institut für Biomedizinische Technik und Informatik), IImenau, Germany systems that allow unrestricted or lateral access $[3,4]$. Therefore, imaging features and image quality metrics may be different in the FOV.

In the beginning of MPI technology development, simple structures such as point samples or filled capillaries were chosen, to test the general performance of MPI scanners $[3,5,6]$. The increasing focus on specific MPI application scenarios has led to the development of more physiological phantoms in recent years. These are designed either as cavities that hold liquid samples with suspended MNP [7-10] or as solids (e.g. gels, polymers, proteins, or sugar matrix) with embedded MNP [11-14].

All MPI phantoms available so far are adapted in size and functionality to the respective MPI scanner geometry and the specific application. Presently, this impedes the harmonization of the various MPI systems. In addition, the defined and homogeneous distribution of MNPs as well as the long-term stability with respect to shape and magnetism still represent a major challenge in phantoms $[11,13]$.

We recently developed a novel process for the additive manufacturing (AM) of parts made of polymer-MNP composites with defined magnetic properties. The parts produced in this way stand out by their homogeneous MNP distribution and long-term stability [15].

In this study we develop and validate a modular MPI phantom kit with high versatility for platform-independent quality assurance and the assembling of defined geometries in MPI. To this end, we investigated the imaging properties of the fabricated single units and an assembled phantom. Reliability for adequate image quality assessment was demonstrated using physiological geometries.

\section{Materials and Methods}

\subsection{Phantom Manufacturing}

The base material used for the modular MPI phantom kit is a light-curing photopolymer resin (R05 red, EnvisionTEC) that is suitable for AM. To produce magnetic components, MNP (EFH3, FerroTec) were added to the base material using an ultrasound-based mixing process. This novel polymer-MNP 
composite is suitable for AM [15]. The phantom parts were manufactured using a Digital Light Processing 3D printing system (Perfactory DSP XL, EnvisionTEC). UV-curing with a UVA-Cube 2000 (Dr. Hönle AG, UV-Technologie) was performed to achieve a complete polymerization, improve layer adhesion and reduce anisotropic properties.

\subsection{Phantom kit design}

The basic concept of the MPI modular phantom kit is a flexible modular structure with the smallest units consisting of a voxelsized cube (size S: $3 \times 3 \times 3 \mathrm{~mm}$ and size L: $6 \times 6 \times 6 \mathrm{~mm}$; see figure 1). The individual units are fabricated either from nonmagnetic polymer or novel polymer-MNP composite using 3D printing [15]. The setup is designed to build reproducible and defined magnetic structures of arbitrary shape (voxel size $>3$ x 3 x $3 \mathrm{~mm}$ ). The cubes were designed with a wave-shape contour (see figure 2), which allows a displacement-safe positioning to each other. Using a support with square top and bottom plate ( $36.4 \mathrm{~mm} \times 36.4 \mathrm{~mm}$ ) with matching wave-shape contour allows the fixed combination of several levels of $12 \mathrm{x}$ 12 cubes (size $\mathrm{S}$ ) into one module using M2 screws (figure 1). The height of the modules can be varied so that the phantoms can be adapted to the respective MPI scanner dimensions. a

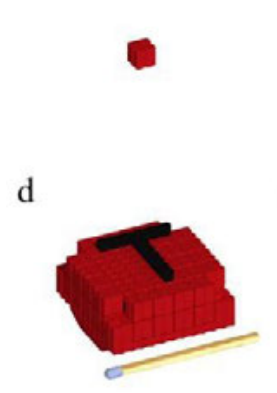

b

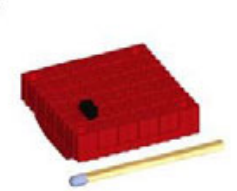

$\mathrm{e}$

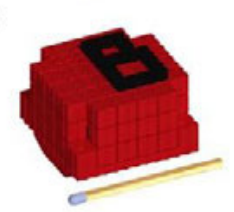

$\mathrm{c}$

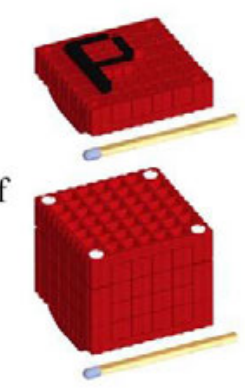

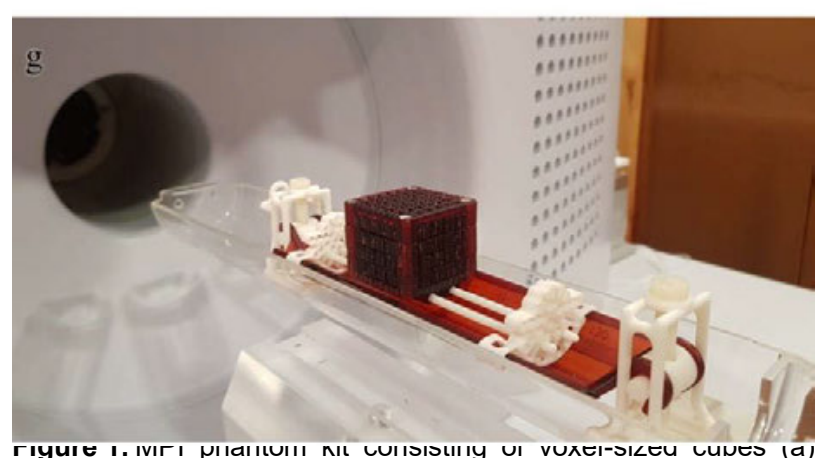
assembled on a base plate (b). By using MPI-visible magnetic cubes (black), magnetic shapes can be created layer by layer (ce). By screwing the top and bottom plates, the MPI phantom kit is created (f) and can be used for MPI experiments ( $g$ ).
Moreover, the module created in this way can be extended by additional modules of the same dimensions. We determined reproducibility and dimensional accuracy of the geometries and estimated an edge length variation of less than $1 \%$.

\subsection{Magnetic Particle Spectroscopy}

The magnetic properties of each voxel-sized cube were determined using high-sensitivity magnetic particle spectroscopy (MPS) which is considered as 0-dimensional MPI. In MPS a sinusoidal excitation field of $B=25 \mathrm{mT}$ amplitude at a frequency $f_{0}=25 \mathrm{kHz}$ was applied to the cubes containing MNP. Due to their non-linear magnetization, the measured response of the MNP contains higher odd harmonics of $f_{0}$ (i.e. $\left.(2 \cdot \mathrm{n}-1) \cdot f_{0}\right)$ in the Fourier transformed spectrum $A_{\mathrm{n}}$. The harmonic amplitudes $A_{\mathrm{n}}$ are directly proportional to the absolute MNP content. Since $A_{3}$ provides the highest signal, it is used for reference sample based quantification of MNP [16]. In addition, the concentration independent ratio $A_{5} / A_{3}$ was determined, which is a measure of the decay of harmonic amplitudes at higher frequencies and is constant for the same MNP type. A flat spectrum, i.e. a high $A_{5} / A_{3}$, indicates a good MPI performance.

\subsection{Magnetic Particle Imaging}

We used a commercial preclinical MPI scanner (MPI 25/20 FF Bruker BioSpin) to analyze the imaging performance of our phantoms [17]. This system is a field free point scanner where we used the system function approach for image reconstruction. Field gradients of $2.5 \mathrm{~T} / \mathrm{m}$ in z-, and $1.25 \mathrm{~T} / \mathrm{m}$ in $\mathrm{x}$ and $\mathrm{y}$ were used for spatial coding.

\section{Results}

\subsection{Magnetic characterization}

The 3D printed cubes (30 pieces per print job) were measured by MPS to analyse the variation of magnetic properties and to determine the suitability for MPI imaging.

We observed a relative variation of less than $3 \%$ in the MNP content directly proportional to the MPS signal amplitude $A_{3}$ (see Tab. 1), reflecting the high reproducibility of the fabrication process. Of note is the high $A_{5} / A_{3}$ value of $21 \%$ of the magnetic cubes, which is close to that of suitable MPI 
Table 1: MPS measurement results for 3D printed magnetic cubes of different size $(\mathrm{S}, \mathrm{L})$ and iron concentration $c(\mathrm{Fe})$. While the MPS signal for non-magnetic cubes was below the detection limit of the MPS $\left(0.03 \mathrm{nAm}{ }^{2}\right)$, high signal amplitudes $A_{3}$ were measured for magnetic cubes. The signal amplitude $A_{3}$ was also normalized to the sample volume $V$. In contrast, the concentration-independent parameter $A_{5} / A_{3}$ remained unchanged. The numbers in parentheses denote the uncertainty of the last digit, e.g. $21.6(2) \%$ reads as $21.6 \pm 0.2 \%$.

\begin{tabular}{|c|c|c|c|c|c|c|}
\hline & \multicolumn{2}{|c|}{ size S } & \multirow[b]{2}{*}{$A_{5} / A_{3}(\%)$} & \multicolumn{2}{|r|}{ size L } & \multirow[b]{2}{*}{$A_{5} / A_{3}(\%)$} \\
\hline & $A_{3}\left(\mathrm{nAm}^{2}\right)$ & $A_{3} / V(\mathrm{~A} / \mathrm{m})$ & & $A_{3}\left(\mathrm{nAm}^{2}\right)$ & $A_{3} / V(\mathrm{~A} / \mathrm{m})$ & \\
\hline non-magnetic ( 1 job, $n=30$ ) & $0.007(3)$ & $0,0003(1)$ & - & $0.009(8)$ & $0,00005(4)$ & - \\
\hline magnetic $(c(\mathrm{Fe})=4 \mathrm{~g} / \mathrm{L}, 1$ job, $\mathrm{n}=30)$ & $164(4)$ & $6,1(1)$ & $21.6(2)$ & $1180(18)$ & $6,37(9)$ & $21.08(9)$ \\
\hline magnetic $(\mathrm{c}(\mathrm{Fe})=3 \mathrm{~g} / \mathrm{L}, 1$ job, $\mathrm{n}=30)$ & $119(3)$ & $4,4(1)$ & $21.7(2)$ & $839(13)$ & $4,53(7)$ & $20.69(8)$ \\
\hline magnetic (low $\mathrm{c}(\mathrm{Fe}), 3$ jobs, $\mathrm{n}=90$ ) & $120(4)$ & $4,4(1)$ & $21.7(2)$ & $834(11)$ & $4,50(5)$ & $20.72(7)$ \\
\hline
\end{tabular}

tracers in immobilized state (e.g. Resovist ${ }^{\circledR} A_{5} / A_{3}=27 \%$ ). A relative deviation of $<1 \%$ over several print jobs and different sizes (size S, size L) underpins the high reproducibility of the overall manufacturing process.

Since the MPS signal amplitude $A_{3}$, in addition to the magnetic properties of the MNP, also depends on the MNP amount, the variation is probably larger than for the mass independent $A_{5} / A_{3}$ ratio. In particular, the incomplete removal of support structures (after 3D printing) leads to an undesired variation in the mass of the printed cubes. Nevertheless, the signal amplitude of each cube can be determined with high accuracy using MPS to create a voxel-wise calibrated phantom.

\subsection{Magnetic Particle Imaging (MPI)}

Finally, the capability of the MPI phantom kit was tested in various application scenarios. First, a defined gradient was investigated. For this purpose, cubes (size S) with different

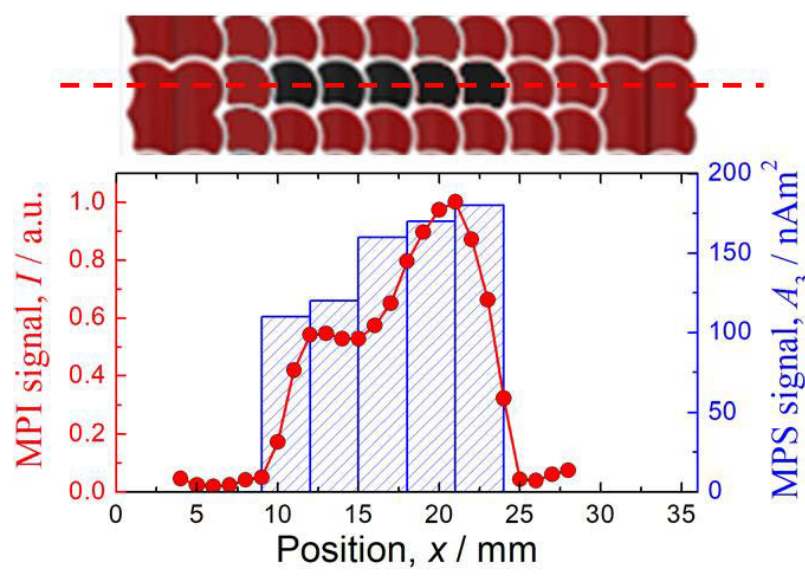

Figure 3: MPI phantom kit consisting of five magnetic cubes (black) with increasing MNP-concentration from left to right. The MPS signal amplitude of each cube (size $S$ ) was measured in advance (blue bars). Shown is the z-projection of the MPI signal intensity (red dashed line) of the reconstructed images (red circles).
MNP concentrations were prepared and the signal amplitude $A_{3}$ was determined using MPS. Subsequently, the cubes were sorted according to their MPS signal amplitude, arranged side by side in the MPI phantom kit and measured with MPI. The reconstructed signal amplitude in MPI is shown in Fig. 2. It can be seen that in this experiment it was not possible to resolve the concentration gradient with very high accuracy. Only the pronounced concentration step of $20 \%$ (cube three to four) is clearly visible in MPI.

In a second experiment, the physiological structure of a bifurcation was created (Fig. 3). For this purpose, cubes with the same MNP concentration $(4 \mathrm{~g}(\mathrm{Fe}) / \mathrm{L})$ were used. In the reconstructed MPI images, the bifurcation is visible. Likewise, the left vessel after bifurcation appears larger in the MPI image, as it is composed of seven cubes (right vessel only six cubes).
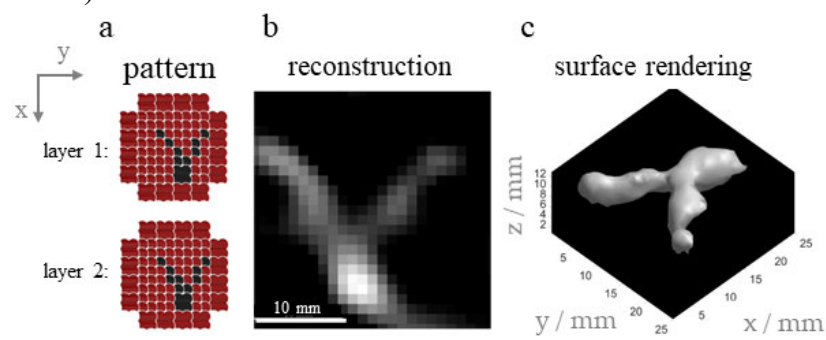

Figure 2: MPI phantom kit consisting of magnetic cubes of equal MNP concentration arranged in the shape of a bifurcation (a). From the signal intensity of the reconstructed MPI image, the created structure can be seen (b), which is also evident in the surface rendering (c).

\section{Conclusion}

We developed and validated a versatile and customizable MPI phantom kit comprising specific requirements for MPI. It was demonstrated that the MPI phantom kit facilitates both, application scenario testing and quality assurance in MPI. An advantage of the setup is that the magnetic properties of the 
individual building blocks can be measured by MPS before a phantom experiment providing a voxel-wise calibrated phantom. Furthermore, this approach enables the comprehensive assessment of MPI image quality with broad versatility. Determination of spatial resolution, comprehensive image quality evaluation, including anatomical replications (voxel size $>3 \times 3 \times 3 \mathrm{~mm}$ ), and quantification accuracy assessment become possible with the novel MPI phantom kit. To better assess the technical limitations of MPI imaging, we aim to embed higher quality MPI tracers into the photopolymers.

In the future, the provision of such reference phantoms should allow direct comparison of existing scanners, leading to harmonization of measurement devices for the MPI community.

\section{Author Statement}

This project was by funded by the Deutsche Forschungsgemeinschaft (DFG, German Research Foundation) within the research grant "CellMPI" (455706279) and "QuantMPI" (DU1293/6-1 and TR408/9-1). Authors state no conflict of interest. Informed consent has been obtained from all individuals included in this study.

\section{References}

[1] Gleich B, Weizenecker J. Tomographic imaging using the nonlinear response of magnetic particles. Nature 2005;435:1214-7. https://doi.org/10.1038/nature03808.

[2] Talebloo N, Gudi M, Robertson N, Wang P. Magnetic Particle Imaging: Current Applications in Biomedical Research. J Magn Reson Imaging 2020;51:1659-68. https://doi.org/10.1002/jmri.26875.

[3] Sattel TF, Knopp T, Biederer S, Gleich B, Weizenecker J, Borgert J, et al. Single-sided device for magnetic particle imaging. J Phys D: Appl Phys 2008;42:022001. https://doi.org/10.1088/0022-3727/42/2/022001.

[4] Pagan J, McDonough C, Vo T, Tonyushkin A. SingleSided Magnetic Particle Imaging Device With FieldFree-Line Geometry for In Vivo Imaging Applications. IEEE Trans Magn 2021;57:1-5. https://doi.org/10.1109/TMAG.2020.3008596.

[5] Goodwill PW, Conolly SM. Multidimensional X-Space Magnetic Particle Imaging. IEEE Trans Med Imaging 2011;30:1581-90. https://doi.org/10.1109/TMI.2011.2125982.

[6] Ferguson RM, Khandhar AP, Kemp SJ, Arami H, Saritas EU, Croft LR, et al. Magnetic Particle Imaging With Tailored Iron Oxide Nanoparticle Tracers. IEEE Trans Med Imaging 2015;34:1077-84. https://doi.org/10.1109/TMI.2014.2375065.
[7] Lu K, Goodwill PW, Saritas EU, Zheng B, Conolly SM. Linearity and Shift Invariance for Quantitative Magnetic Particle Imaging. IEEE Trans Med Imaging 2013;32:10.1109/TMI.2013.2257177. https://doi.org/10.1109/TMI.2013.2257177.

[8] Graeser M, Thieben F, Szwargulski P, Werner F, Gdaniec $\mathrm{N}$, Boberg $\mathrm{M}$, et al. Human-sized magnetic particle imaging for brain applications. Nat Commun 2019;10:1936. https://doi.org/10.1038/s41467-01909704-x.

[9] Exner M, Szwargulski P, Knopp T, Graeser M, Ludewig P. 3D Printed Anatomical Model of a Rat for Medical Imaging. Curr Dir Biomed 2019;5:187-90. https://doi.org/10.1515/cdbme-2019-0048.

[10] Dietrich P, Vogel P, Kampf T, Rückert MA, Behr VC, Bley TA, et al. Near real-time magnetic particle imaging for visual assessment of vascular stenosis in a phantom model. Physica Medica 2021;81:210-4. https://doi.org/10.1016/j.ejmp.2020.12.020.

[11] Mattern A, Sandig R, Joos A, Löwa N, Kosch O, Weidner A, et al. Magnetic Nanoparticle-Gel Materials for Development of MPI and MRI Phantoms. Int J Magn Part Imaging 2018;4 https://doi.org/10.18416/IJMPI.2018.1811001.

[12] Salamon J, Dieckhoff J, Kaul MG, Jung C, Adam G, Möddel M, et al. Visualization of spatial and temporal temperature distributions with magnetic particle imaging for liver tumor ablation therapy. Sci Rep 2020;10:7480. https://doi.org/10.1038/s41598-020-64280-1.

[13] Wöckel L, Wells J, Kosch O, Lyer S, Alexiou C, Grüttner $\mathrm{C}$, et al. Long-term stable measurement phantoms for magnetic particle imaging. J Magn Magn Mater 2019;471:1-7. https://doi.org/10.1016/j.jmmm.2018.09.012.

[14] Kosch O, Paysen H, Wells J, Ptach F, Franke J, Wöckel $\mathrm{L}$, et al. Evaluation of a separate-receive coil by magnetic particle imaging of a solid phantom. J Magn Magn Mater 2019;471:444-9. https://doi.org/10.1016/j.jmmm.2018.09.114.

[15] Löwa N, Fabert J-M, Gutkelch D, Paysen H, Kosch O, Wiekhorst F. 3D-printing of novel magnetic composites based on magnetic nanoparticles and photopolymers. J Magn Magn Mater 2019;469:456-60. https://doi.org/10.1016/j.jmmm.2018.08.073.

[16] Löwa N, Wiekhorst F, Gemeinhardt I, Ebert M, Schnorr J, Wagner S, et al. Cellular Uptake of Magnetic Nanoparticles Quantified by Magnetic Particle Spectroscopy. IEEE Trans Magn 2013;49:275-8. https://doi.org/10.1109/TMAG.2012.2218223.

[17] Magnetic Particle Imaging | MPI System | Preclinical n.d. https://www.bruker.com/en/products-andsolutions/preclinical-imaging/mpi.html (accessed September 6, 2021). 